\title{
Approaching zero: temporal effects of a restrictive antibiotic policy on hospital-acquired Clostridium difficile, extended-spectrum $\beta$-lactamase-producing coliforms and meticillin-resistant Staphylococcus aureus
}

\author{
S.J. Dancer ${ }^{\mathrm{a}, *}$, P. Kirkpatrick ${ }^{\mathrm{a}}$, D.S. Corcoran ${ }^{\mathrm{b}}$, F. Christison ${ }^{\mathrm{a}}$, D. Farmer ${ }^{\mathrm{c}}$, C. Robertson ${ }^{\mathrm{d}, \mathrm{e}, \mathrm{f}}$ \\ a Department of Microbiology, Hairmyres Hospital, East Kilbride, Lanarkshire G75 8RG, UK \\ ${ }^{\mathrm{b}}$ Department of Medicine, Hairmyres Hospital, East Kilbride, Lanarkshire G75 8RG, UK \\ c Department of Pharmacy, Hairmyres Hospital, East Kilbride, Lanarkshire G75 8RG, UK \\ ${ }^{\mathrm{d}}$ Department of Mathematics $\mathcal{E}$ Statistics, University of Strathclyde, Glasgow G1 1XH, UK \\ e Health Protection Scotland, Glasgow, UK \\ ${ }^{\mathrm{f}}$ International Prevention Research Institute, Lyon, France
}

\section{A R T I C L E I N F O}

\section{Article history:}

Received 16 July 2012

Accepted 26 October 2012

\section{Keywords:}

Antimicrobial stewardship

Clostridium difficile

MRSA

ESBL-producing coliforms

Hospital-acquired infection

\begin{abstract}
A B S T R A C T
A restrictive antibiotic policy banning routine use of ceftriaxone and ciprofloxacin was implemented in a 450-bed district general hospital following an educational campaign. Monthly consumption of nine antibiotics was monitored in defined daily doses (DDDs) per 1000 patient-occupied bed-days (1000 pt-bds) 9 months before until 16 months after policy introduction. Hospital-acquired Clostridium difficile, meticillin-resistant Staphylococcus aureus (MRSA) and extended-spectrum $\beta$-lactamase (ESBL)producing coliform cases per month/1000 pt-bds were identified and reviewed throughout the hospital. Between the first and final 6 months of the study, average monthly consumption of ceftriaxone reduced by $95 \%$ (from 46.213 to $2.129 \mathrm{DDDs} / 1000$ pt-bds) and that for ciprofloxacin by $72.5 \%$ (109.804 to 30.205 DDDs/1000 pt-bds). Over the same periods, hospital-acquisition rates for $C$. difficile reduced by $77 \%$ (2.398 to 0.549 cases $/ 1000$ pt-bds), for MRSA by $25 \%$ ( 1.187 to 0.894 cases/1000 pt-bds) and for ESBL-producing coliforms by $17 \%$ ( 1.480 to 1.224 cases/1000 pt-bds). Time-lag modelling confirmed significant associations between ceftriaxone and $C$. difficile cases at 1 month (correlation $0.83 ; P<0.005$ ), and between ciprofloxacin and ESBL-producing coliform cases at 2 months (correlation $0.649 ; P=0.002$ ). An audit performed 3 years after the policy showed sustained reduction in $C$. difficile rates $(0.259$ cases/1000 pt-bds), with additional decreases for MRSA (0.409 cases/1000 pt-bds) and ESBL-producing coliforms ( 0.809 cases $/ 1000 \mathrm{pt}$-bds). In conclusion, banning two antibiotics resulted in an immediate and profound reduction in hospital-acquired C. difficile, with possible longer-term effects on MRSA and ESBL-producing coliform rates. Antibiotic stewardship is fundamental in the control of major hospital pathogens.
\end{abstract}

(c) 2012 Elsevier B.V. and the International Society of Chemotherapy. All rights reserved.

\section{Introduction}

There are continuing concerns over hospital-acquired infections (HAIs) caused by Clostridium difficile, meticillin-resistant Staphylococcus aureus (MRSA) and extended-spectrum $\beta$-lactamase (ESBL)-producing coliforms [1]. It is already known that these pathogens are associated with antibiotic consumption, with some classes encouraging certain organisms more than others [1-3]. Intensive use of cephalosporins or quinolones, for example, has been linked with increasing rates of $C$. difficile, MRSA and/or ESBLproducing coliforms, whilst reduced consumption has facilitated

\footnotetext{
* Corresponding author. Tel.: +44 1355585 000; fax: +44 1355584350 .

E-mail address: stephanie.dancer@lanarkshire.scot.nhs.uk (S.J. Dancer).
}

decreased rates of one or more of these pathogens [1-5]. As a response to high rates of HAIs, it was decided to impose a restrictive antibiotic policy banning the routine use of third-generation cephalosporins, specifically ceftriaxone, and quinolones throughout the hospital. Prospective surveillance programmes were set up to monitor rates of hospital-acquired $C$. difficile, MRSA and ESBL-producing coliforms. Previous studies have not necessarily monitored the effect of such a policy for a whole hospital, nor have they included surveillance of all three pathogens simultaneously. The aim of stewardship was primarily to reduce the number of C. difficile cases, although it was hoped that the policy would also impact on MRSA and ESBL-producing coliforms. This article describes how prescribing practices were changed and what happened after two key antibiotics were removed from first-line prescribing on a hospital-wide basis. 


\section{Materials and methods}

\subsection{Antimicrobial policy implementation}

Hairmyres Hospital is a 450-bed district general hospital in a rural area just outside Glasgow, UK. The hospital admits adult patients only, specialising in care of the elderly, respiratory, endocrinology and cardiology, with most surgical specialties represented, including orthopaedics, vascular, ear, nose and throat (ENT), and ophthalmology. There is an Accident \& Emergency (A\&E) department, a high dependency unit and an 8-bed Intensive Care Unit (ICU). Antimicrobial prescribing policies for empirical treatment of various infections and surgical prophylaxis are overseen by a Healthboard Infection Committee; day-to-day advice on antimicrobial prescribing is provided by one consultant microbiologist, who also authorises microbiological results. The microbiologist is supported by an on-site Clinical Pathology Accreditation (CPA)-accredited laboratory, out-of-hours cross cover by colleagues at neighbouring hospitals and two infection control nurses.

Specific concern over $C$. difficile was raised following increasing cases among hospital in-patients during January 2008. Surveillance figures published later that year confirmed the high rate of $C$. difficile compared with other Scottish hospitals [6]. It was decided to initiate an educational programme encouraging prescribers to reduce consumption of cephalosporins and quinolones on a voluntary basis. This was done by providing a series of lectures to all medical staff starting in January 2008 as well as weekly teaching for small groups of junior doctors. A letter summarising the problem and requirement for prescribing change was sent to all senior staff, including doctors, pharmacists and managers. Daily microbiological authorisation suppressed release of cephalosporins and quinolones on ward reports unless no other agents were available. Ad hoc discussion took place when ward doctors phoned for advice on individual patients, with restricted agents either stopped or changed to alternative therapy.

From January 2008, the infection control team initiated prospective surveillance of all cases of $C$. difficile, MRSA and ESBL-producing coliforms on a ward-by-ward basis using national guidelines and definitions. Monthly consumption of antibiotics was collected and recorded in defined daily doses (DDDs) per 1000 patient-occupied bed-days (pt-bds) (http://www.whocc.no/atc_ddd_index/). Macrolides (erythromycin and clarithromycin), ceftriaxone, amoxicillin, amoxicillin/clavulanic acid, piperacillin/tazobactam, gentamicin, ciprofloxacin and meropenem were monitored. Information Management provided monthly bed occupancy figures for the hospital using the hospital TrakCare ${ }^{\circledR}$ system.

Following a small outbreak of $C$. difficile on the gastroenterology ward in May 2008, formal restriction of cephalosporins and quinolones was proposed. This began during August 2008, to coincide with hospital-wide changeover of junior doctors. The antimicrobial policy was contained within an induction programme for all new doctors. Empirical prescription of ceftriaxone for systemic sepsis and surgical prophylaxis was changed to amoxicillin, gentamicin and metronidazole. The most notable intervention was complete removal of ceftriaxone and ciprofloxacin from all wards except for A\&E and the ICU. The educational programme then continued for prescribers on a regular basis, with feedback on HAI rates sent to clinicians and managers from late 2008. The antibiotic policy continued after the study finished in December 2009.

Preliminary data from this study were presented at the 20th European Congress of Clinical Microbiology and Infectious Diseases (ECCMID), 10-13 April 2010, Vienna, Austria. One year later, 2011 rates of $C$. difficile, MRSA and ESBL-producing coliforms were audited using the same definitions in order to gauge long-term effects of the restrictive policy. HAI and bed occupancy data were subsequently collected on a monthly basis throughout 2011.

\subsection{Surveillance definitions}

Specimens were sent to the laboratory when clinically indicated. Hospital-acquired $C$. difficile was defined as onset of symptoms $>48 \mathrm{~h}$ after admission (or with onset of symptoms in the community following hospital admission within the previous 4 weeks) along with a positive $C$. difficile toxin test. Hospital-acquired MRSA was confirmed if MRSA was isolated from any specimen $>48 \mathrm{~h}$ after hospital admission, with no previous record of MRSA carriage or infection. Similarly, ESBL-producing coliform isolated from any specimen $>48 \mathrm{~h}$ after admission was defined as hospital-acquired, with no previous record of ESBL-producing coliform carriage or infection. Whilst screening for these organisms was not routinely performed during 2007-2009, patients awaiting elective surgery were offered MRSA screening (nose, perineum and wounds) and urinalysis.

Determination of new acquisitions was performed systematically for all patients following laboratory confirmation. After excluding duplicate specimens, each new acquisition alert was subjected to rigorous investigation, including inspection of patient notes, details on previous hospital admission(s), antimicrobial therapy and review of microbiological history using the laboratory database. Whilst new acquisitions of $C$. difficile were generally conclusive, investigation of potential new acquisitions of MRSA and ESBL-producing coliforms often revealed prior acquisition months or even years before the current admission and these patients were excluded. There were no genotyping data to confirm persistent carriage of specific strains, but the database held details of previously isolated organisms with full antibiogram. This helped evaluate long-term carriage of specific phenotypes of MRSA and ESBL-producing coliforms. Microbiological results for individual patients moving between hospital and the community could be tracked for a decade or more.

Patients lacking historical microbiological data and/or history of recent (within 1 year) hospital admission and/or transfers from another hospital or healthcare institution were excluded, as well as frequent hospital attendees due to chronic disease. Patient notes were tagged for future reference following confirmation of acquisition. Enhanced investigation of all potential acquisitions was maintained throughout the study owing to the fact that patients were not routinely screened for study organisms. Universal screening for MRSA was introduced as national policy in 2010.

Healthboard policies were applied throughout the whole hospital by the same infection control team. Other than sporadic ward closures due to norovirus, there were no outbreaks caused by any of the three organisms under surveillance, apart from the cluster of $C$. difficile cases previously mentioned, and there were no additional infection control interventions over the study period.

\subsection{Laboratory methods}

Isolation and identification of $C$. difficile, MRSA and ESBLproducing coliforms from patient specimens were subject to standard operating procedures in an accredited NHS microbiology laboratory. Briefly, $C$. difficile in liquid stool was confirmed by enzyme immunoassay assay ( $C$. difficile Tox A/B II; TechLab Inc.), with a proportion of cultured isolates sent to a reference facility for further characterisation. Selection of isolates for typing adhered to reference laboratory recommendations, e.g. investigation of case clusters, fatal outcome and multiple occurrences.

Specimens from all sites were cultured on Oxoid Brilliance Chromogenic MRSA agar overnight and suspect colonies were checked 
Table 1

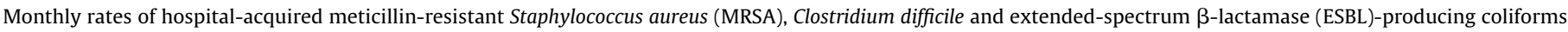
and consumption of key antibiotics following introduction of a restrictive antibiotic policy for 2 years.

\begin{tabular}{|c|c|c|c|c|c|c|c|c|c|c|c|}
\hline \multirow[t]{2}{*}{ Date } & \multicolumn{3}{|c|}{ Rate (/1000 pt-bds) } & \multicolumn{8}{|c|}{ Consumption (DDDs/1000 pt-bds) } \\
\hline & MRSA & C. difficile & ESBL-producing coliforms & CFX & GEN & TZP & AMX & AMC & Macro & CIP & MER \\
\hline Nov. 2007 & $\mathrm{n} / \mathrm{c}$ & $\mathrm{n} / \mathrm{c}$ & 1.220 & 58.713 & 19.068 & 12.210 & 144.579 & 274.5 & 35.64 & 51.68 & 13.834 \\
\hline Dec. 2007 & $\mathrm{n} / \mathrm{c}$ & 1.883 & 1.130 & 83.254 & 24.800 & 21.705 & 223.582 & 363.147 & 39.27 & 96.571 & 8.005 \\
\hline Jan. 2008 & 2.078 & 3.03 & 1.212 & 70.515 & 27.991 & 12.690 & 131.861 & 272.06 & 43.74 & 87.654 & 4.545 \\
\hline Feb. 2008 & 0.735 & 1.837 & 1.377 & 44.219 & 21.122 & 11.601 & 160.139 & 336.909 & 30.83 & 96.905 & 8.265 \\
\hline Mar. 2008 & 1.081 & 2.972 & 1.171 & 54.535 & 26.867 & 14.533 & 138.363 & 265.243 & 36.90 & 52.526 & 7.881 \\
\hline Apr. 2008 & 1.130 & 1.977 & 1.695 & 41.612 & 44.879 & 15.191 & 141.310 & 146.921 & 33.48 & 142.045 & 12.945 \\
\hline May 2008 & 1.433 & 2.580 & 1.147 & 34.595 & 40.300 & 25.281 & 134.27 & 247.343 & 37.20 & 128.937 & 8.123 \\
\hline June 2008 & 0.664 & 1.993 & 2.278 & 31.800 & 34.333 & 18.428 & 122.662 & 264.233 & 35.64 & 150.755 & 9.255 \\
\hline July 2008 & 0.751 & 0.751 & 1.408 & 27.509 & 41.780 & 19.405 & 162.473 & 215.995 & 32.13 & 146.747 & 7.746 \\
\hline Aug. 2008 & 0.497 & 0.795 & 1.889 & 5.668 & 20.384 & 18.505 & 167.023 & 177.468 & 29.26 & 118.345 & 4.474 \\
\hline Sept. 2008 & 0.932 & 1.553 & 2.795 & 4.347 & 39.675 & 12.21 & 257.711 & 180.944 & 43.50 & 82.124 & 3.364 \\
\hline Oct. 2008 & 0.870 & 0.580 & 1.644 & 1.692 & 39.476 & 12.99 & 259.62 & 153.476 & 44.93 & 57.411 & 2.66 \\
\hline Nov. 2008 & 1.403 & 1.309 & 0.842 & 2.619 & 60.799 & 10.773 & 260.499 & 172.145 & 42.46 & 73.931 & 4.911 \\
\hline Dec. 2008 & 1.082 & 0.541 & 1.442 & 2.884 & 88.33 & 16.139 & 505.858 & 266.805 & 70.03 & 68.265 & 5.858 \\
\hline Jan. 2009 & 0.802 & 0.356 & 0.713 & 1.912 & 80.23 & 12.55 & 271.684 & 140.649 & 47.13 & 105.17 & 4.903 \\
\hline Feb. 2009 & 0.728 & 0.312 & 1.04 & 2.496 & 64.832 & 14.376 & 312.981 & 182.016 & 42.59 & 64.76 & 3.640 \\
\hline Mar. 2009 & 0.738 & 0.277 & 1.845 & 1.015 & 63.653 & 18.295 & 254.336 & 157.300 & 40.27 & 50.387 & 4.612 \\
\hline Apr. 2009 & 1.375 & 0.458 & 0.825 & 2.933 & 56.365 & 18.412 & 193.52 & 118.733 & 43.4 & 10.503 & 5.957 \\
\hline May 2009 & 0.534 & 0.802 & 0.98 & 2.316 & 70.372 & 19.126 & 284.874 & 117.967 & 44.81 & 27.632 & 6.235 \\
\hline June 2009 & 1.359 & 0.291 & 0.874 & 1.310 & 82.343 & 18.533 & 248.932 & 159.02 & 41.62 & 30.072 & 2.184 \\
\hline July 2009 & 0.559 & 0.559 & 1.676 & 4.376 & 80.382 & 25.012 & 364.97 & 141.676 & 29.19 & 28.305 & 7.682 \\
\hline Aug. 2009 & 0.933 & 1.027 & 1.587 & 1.166 & 71.236 & 18.029 & 216.239 & 165.273 & 30.19 & 36.211 & 9.800 \\
\hline Sept. 2009 & 0.865 & 0.288 & 0.865 & 0.576 & 72.220 & 14.920 & 281.013 & 135.62 & 36.27 & 27.308 & 6.966 \\
\hline Oct. 2009 & 0.765 & 0.510 & 1.020 & 3.188 & 76.670 & 14.922 & 345.761 & 102.57 & 37.81 & 26.87 & 2.551 \\
\hline Nov. 2009 & 0.882 & 0.617 & 1.323 & 2.161 & 93.066 & 20.67 & 324.32 & 125.803 & 45.85 & 32.463 & 2.426 \\
\hline
\end{tabular}

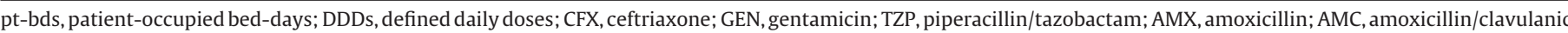
acid; Macro, macrolides (erythromycin and clarithromycin); CIP, ciprofloxacin; MER, meropenem; n/c, not collected.

The educational component of restrictive antibiotic policy began in December 2007; formal component of policy was introduced in July 2008.

for coagulase production using Staph Xtra Latex (Pro-Lab Diagnostics) followed by tube coagulase. Presumed MRSA were tested for penicillin-binding protein using Latex PBP2I (Oxoid Ltd.) and VITEK 2 analyses for confirmation and antibiogram. Sporadic isolates were sent to a reference facility according to national protocol.

ESBL-producing coliforms were also identified using the VITEK 2 analyser, accompanied by standard cephalosporin and clavulanic acid disk testing (Mast Diagnostics). The definition of 'coliforms' used was lactose-fermenting Gram-negative non-spore-forming bacilli capable of producing acid and gas when incubated at $35-37^{\circ} \mathrm{C}$. The following genera and species were identified and included: Escherichia coli; Klebsiella spp. (mostly Klebsiella pneumoniae); Enterobacter spp. (mostly Enterobacter cloacae); Serratia spp. (mostly Serratia marcescens); Hafnia alvei; and Citrobacter koseri. Over 95\% of the ESBL-producing Gram-negative bacilli identified were E. coli and K. pneumoniae. Any unidentifiable isolates and/or those showing resistance to carbapenem agents were sent to a reference laboratory for verification.

\subsection{Statistics}

A Poisson regression model (adjusted for overdispersion) was used with log occupied bed-days as an offset. Linear trend terms were used to assess temporal changes. The main hypothesis was to see whether the trend in HAI rates following antimicrobial restriction was the same as the trend before the intervention. If that hypothesis was not rejected, then there was a further test of the effect of the restrictions in shifting the level of HAI cases. Variable selection was based upon Akaike information criterion (AIC), and residual analysis was used to assess model fit and serial correlation.

Correlations between cases of ESBL-producing coliforms and ciprofloxacin or ceftriaxone (adjusted for patient-occupied beddays) were calculated at time lags of 0-5 months. Poisson regression was again used to assess which of the antibiotics was the better predictor of ESBL-producing coliform cases.

\section{Results}

Average monthly consumption of ceftriaxone reduced from 46.213 DDDs/1000 pt-bds for January-June 2008 to 2.129 DDDs/1000 pt-bds for June-November 2009 (95\% reduction). Over the same periods, ciprofloxacin consumption decreased from 109.804 to 30.205 DDDs/1000 pt-bds (72.5\% reduction). The pre-intervention hospital-acquisition MRSA rate during the initial 6 months was 1.187 cases/1000 pt-bds, decreasing to 0.894 cases/1000 pt-bds (25\% reduction) for the final 6 months of the study; for $C$. difficile it was 2.398 cases/1000 pt-bds, decreasing to 0.549 cases $/ 1000$ pt-bds ( $77 \%$ reduction); and for ESBL-producing coliforms it was 1.480 cases/1000 pt-bds, decreasing to 1.224 cases $/ 1000$ pt-bds ( $17 \%$ reduction).

Prior to formal introduction of the policy in August 2008, the rate of $C$. difficile cases was already decreasing at a rate of $6.59 \%$ per month [95\% confidence interval (CI), $-2.52 \%, 15.02 \%$; $P=0.169$ ] (Table 1; Fig. 1). This was attributed to the educational component of the intervention, which could explain the lack of policy effect on the changing trend of $C$. difficile infections $(P=0.69)$. Adjusting for the decreasing trend, the policy was associated with a $45.22 \%$ reduction (95\% CI, $-4.79 \%, 72.05 \% ; P=0.09$ ) in the rate of $C$. difficile infections. If the trend is ignored, which is reasonable for the data from December 2007 to June 2008, then the policy was associated with a $70.20 \%(95 \% \mathrm{CI}, 57.12 \%, 79.53 \%)$ reduction in the rate of $C$. difficile.

For MRSA, there was no evidence of any effect of the restrictive policy on the trend $(P=0.78)$ nor on the rates of MRSA acquisition following introduction of the policy $(P=0.62)$. The total MRSA acquisition rate was constant over the study period. The MRSA bacteraemia rate in this hospital was initially low 0.4 cases/1000 acute occupied bed-days) and did not change during the study. For ESBLproducing coliforms, there was borderline evidence of an effect of the policy on the trend $(P=0.075)$, which gave an $8.21 \%(95 \% \mathrm{CI}$, $-0.39 \%, 16.15 \%)$ reduction. 


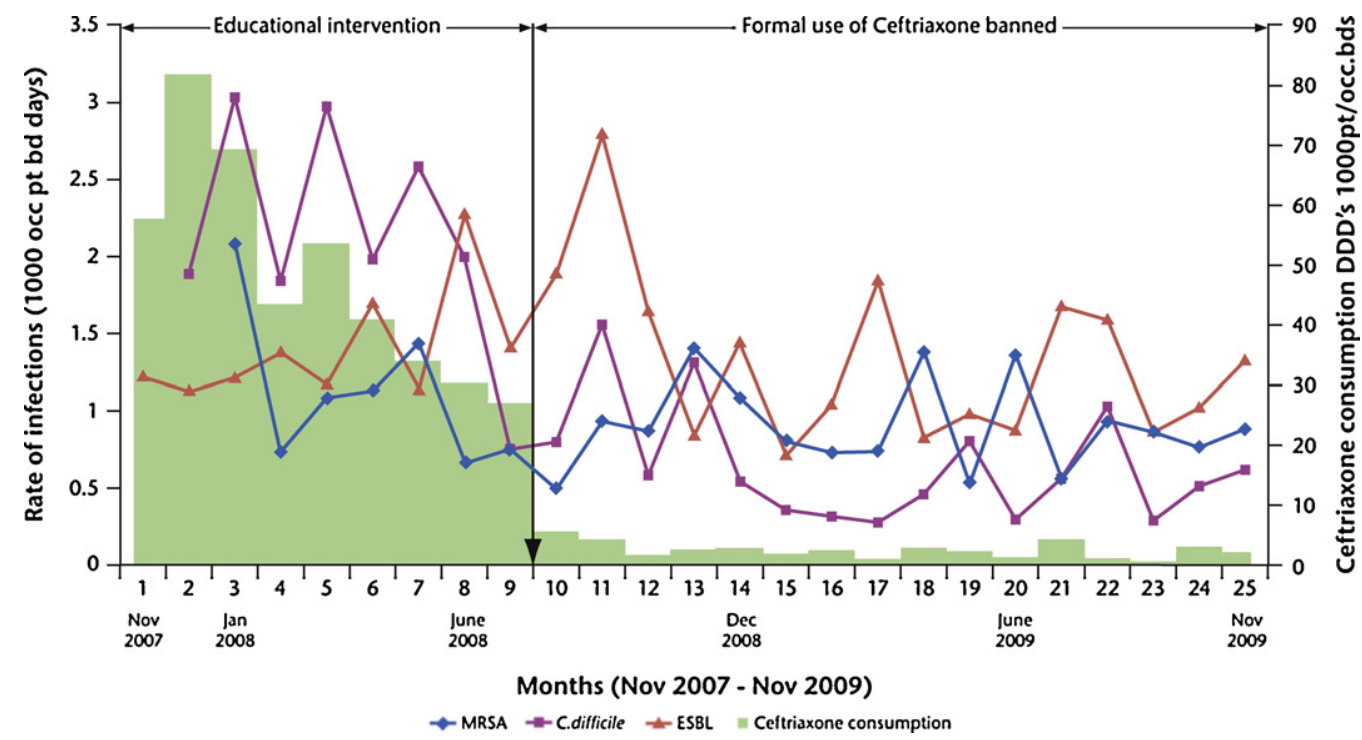

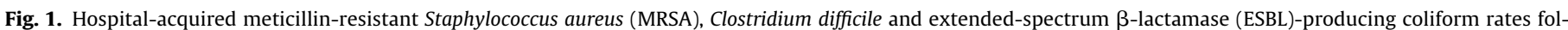
lowing a restrictive antibiotic policy in a district general hospital over 2 years. pt/occ.bds, patient-occupied bed-days; DDDs, defined daily doses.

Based upon AIC, backward selection within the Poisson regression model using an overdispersed distribution showed that the antibiotics most associated with the temporal changes of $C$. difficile were ceftriaxone (rates decrease as ceftriaxone consumption decreases) and amoxicillin (rates decrease as amoxicillin consumption increases) (Table 2). Time-lag modelling showed a 1-month association between ceftriaxone and $C$. difficile cases (correlation $0.83 ; P<0.005)$.

Time-lagged investigation (0-5 months) was used to examine possible associations between ciprofloxacin consumption and ESBL-producing coliform infections (correlation 0.36: $P=0.08$ ) (Table 2; Fig. 2). Adjusting for any association between ceftriaxone and ESBL-producing coliform rates, ciprofloxacin consumption and ESBL-producing coliform rates were associated at lags 0,1 and 2 months (Fig. 3). The strongest association occurred at a lag of 2 months (correlation 0.649; $P=0.002$ ), whilst associations at lags 0 and 1 month are borderline $(P=0.047$ and $P=0.044$, respectively). ESBL-producing coliform rates increased as monthly ciprofloxacin consumption increased for all three lags. Adjusting for the association between ciprofloxacin and ESBL-producing coliform rates, there was no association between ESBL-producing coliforms and ceftriaxone consumption at any lag between 0 and 5 months.

This study finished at the end of 2009, but restrictions on ciprofloxacin and ceftriaxone have continued. Low rates of $C$. difficile have been maintained, as during 2011 monthly rates varied between 0.2 and 0.6 cases $/ 1000$ pt-bds, with an average rate of 0.259 cases/1000 pt-bds (data not shown). We also found decreased numbers of patients acquiring MRSA and ESBL-producing coliforms. In 2011, the average monthly hospitalacquired rate for ESBL-producing coliforms was 0.809 cases/1000 pt-bds and for MRSA it was 0.409 cases/1000 pt-bds, with an MRSA bacteraemia rate of 0.2 cases/1000 acute occupied bed-days. These rates represent further reductions for all three pathogens.

\section{Discussion}

Introducing and reinforcing a restrictive antimicrobial policy in this hospital had a rapid and profound effect on the rate of hospital-acquired $C$. difficile. The relationship between consumption of broad-spectrum antibiotics and $C$. difficile rates has already been established, with the highest risk for $C$. difficile infection within the first month following antibiotic use [1-3,7]. Whilst the most significant association occurred between $C$. difficile rate and ceftriaxone consumption, it is possible that reduction of cases could be attributed to other antibiotic trends. Clostridium difficile isolates were typed and whilst ribotype 0127 was not identified, we were aware of others that were uniformly resistant to quinolone antibiotics. Targeting both ceftriaxone and ciprofloxacin may have ultimately been the most important factor in decreasing $C$. difficile rates.

At the time of this study, there were no national data on antimicrobial consumption for individual hospitals. This was one of the reasons that we decided to examine our infection rates against consumption of key antibiotics. Furthermore, there were no national

Table 2

Correlation between antibiotic consumption and acquisition rate of target pathogens per 1000 patient-occupied bed-days over 2 years in a district general hospital.

\begin{tabular}{|c|c|c|c|c|c|c|}
\hline \multirow[t]{2}{*}{ Antibiotic or antibiotic class } & \multicolumn{2}{|c|}{ Clostridium difficile } & \multicolumn{2}{|l|}{ MRSA } & \multicolumn{2}{|c|}{ ESBL-producing coliforms } \\
\hline & Correlation & $P$-value & Correlation & $P$-value & Correlation & $P$-value \\
\hline Ceftriaxone & 0.83 & $<0.005$ & 0.13 & 0.58 & -0.02 & 0.92 \\
\hline Gentamicin & -0.70 & $<0.005$ & 0.07 & 0.77 & -0.30 & 0.14 \\
\hline Piperacillin/tazobactam & -0.06 & 0.77 & -0.05 & 0.81 & 0.04 & 0.84 \\
\hline Amoxicillin & -0.65 & $<0.005$ & -0.13 & 0.56 & -0.12 & 0.58 \\
\hline Amoxicillin/clavulanic acid & 0.65 & $<0.005$ & 0.06 & 0.78 & 0.11 & 0.61 \\
\hline Macrolides & -0.21 & 0.34 & 0.27 & 0.22 & -0.17 & 0.43 \\
\hline Ciprofloxacin & 0.48 & 0.02 & -0.06 & 0.78 & 0.36 & 0.08 \\
\hline Meropenem & 0.46 & 0.03 & 0.04 & 0.86 & 0.09 & 0.68 \\
\hline
\end{tabular}

MRSA, meticillin-resistant Staphylococcus aureus; ESBL, extended-spectrum $\beta$-lactamase. 


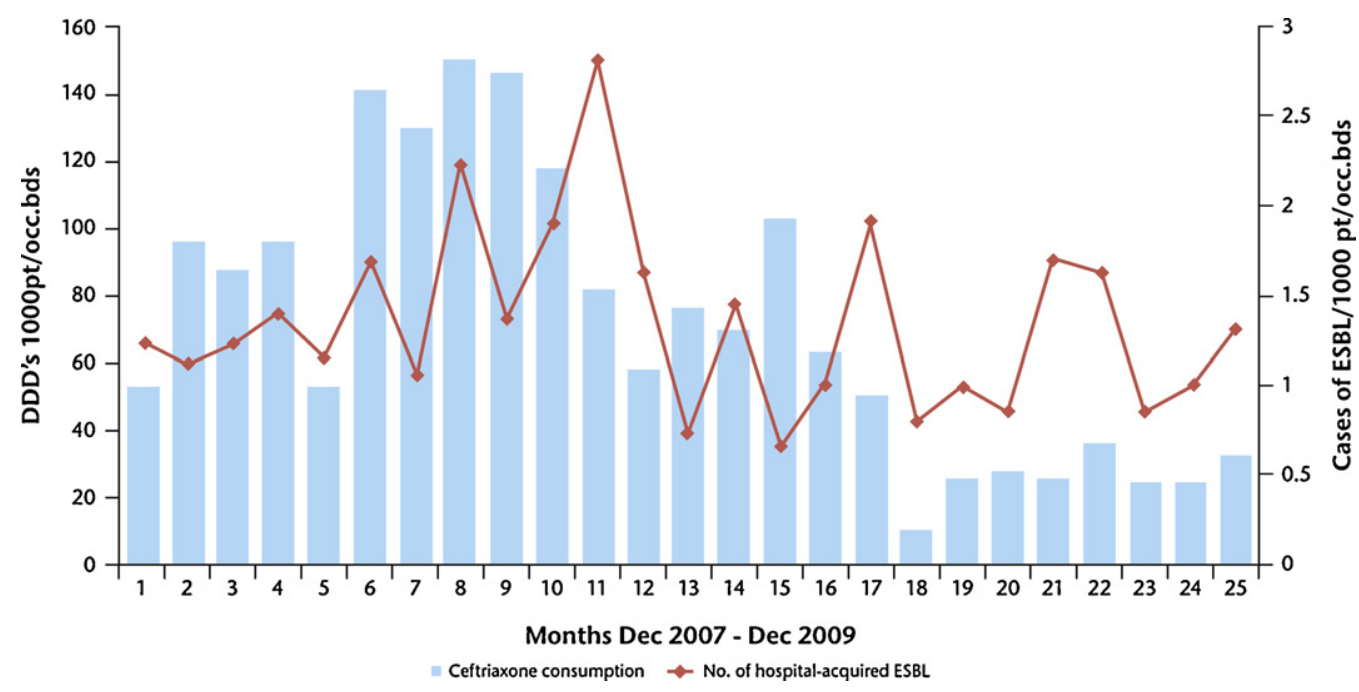

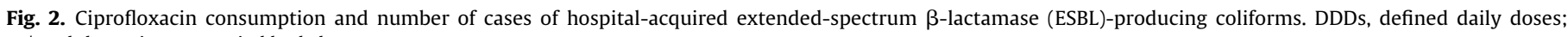
pt/occ.bds, patient-occupied bed-days.

data on ESBL-producing coliform rates, although we knew that the MRSA bacteraemia rate was comparable with other Scottish hospitals of similar size.

Spatiotemporal modelling suggests that reducing patient susceptibility to $C$. difficile infection is more effective for control than trying to stop transmission once a patient is infected [8]. This is supported by our experience and contrasts with the finding that there was little or no effect on initial rates of MRSA or ESBL-producing coliforms [9]. Infection control procedures for $C$. difficile may be more effective for preventing $C$. difficile transmission than those implemented for patients with either MRSA or ESBL-producing coliforms. Patients with diarrhoea were prioritised for isolation, whereas those colonised with MRSA or ESBL-producing coliforms were not. Whilst some ESBL-producing coliform strains can be maintained in humans without any identifiable selective pressure,

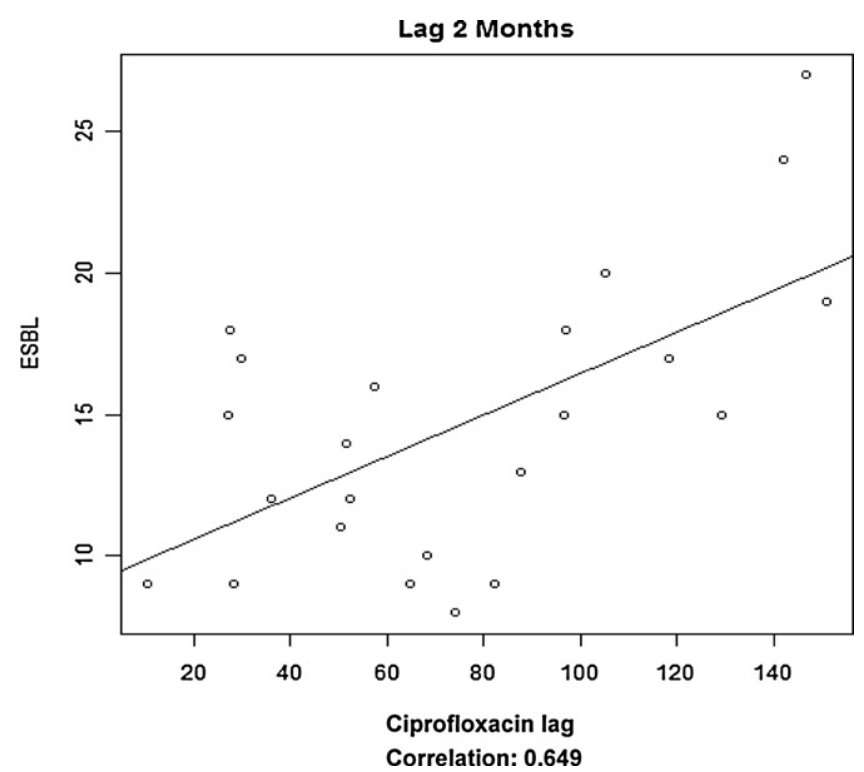

Fig. 3. Time-lag modelling of ciprofloxacin consumption against extendedspectrum $\beta$-lactamase (ESBL)-producing coliform acquisition rates. Adjusting for any association between ceftriaxone and ESBL-producing coliform rates, there is an association between ciprofloxacin usage and ESBL-producing coliforms at lags 0, 1 and 2 months. The strongest association is at a lag of 2 months $(P=0.002)$; associations at lags 0 and 1 month are borderline $(P=0.047$ and $P=0.044$, respectively). we found a time-lag relationship between ciprofloxacin consumption and the incidence of ESBL-producing coliforms [1]. It is possible that ciprofloxacin use is driving rates of ESBL-producing coliforms on a hospital-wide basis. This is supported by two studies showing that fluoroquinolone use correlates with increased ESBL-producing coliforms in ICU patients, and another linking quinolone use with ESBL-producing coliforms in hospital and the community. The latter found a 1-month lag effect between quinolone consumption and ESBL-producing coliform rates rather than the 2 months reported here [10-12].

Gaining support for restricting antibiotics among prescribing colleagues in the hospital initially proved difficult. The educational component was almost exclusively provided by the on-site microbiologist and helped reinforce knowledge on prescribing practices and the longer-term consequences of antibiotic consumption $[2,13]$. The effect of the initial education programme is clearly seen in Fig. 1, whereby consumption of ceftriaxone decreased even before the restrictive policy was formally implemented. Advice on prescribing often conflicted with published guidelines on the management of a specific condition, however, which recommended one or both of the targeted antibiotics. Thus, gastroenterologists initially refused to curtail ciprofloxacin prescribing for spontaneous bacterial peritonitis (SBP) [14]. Restrictions were eventually accepted after the outbreak in May 2008, following which independent audit was performed on the microbiological aetiology of SBP in order to improve prescribing practices. By far the best method of restricting use of a particular drug was physical removal from ward stores by the pharmacists [15]. This meant that doctors did not have easy access to banned agents out-of-hours, although pharmacy would supply it on an individual named-patient basis following clinical discussion. Routine surveillance confirmed that unrestricted antibiotic prescribing did not encourage ICU-acquired infection caused by MRSA, ESBL-producing coliforms or $C$. difficile, but it is possible that daily ward rounds by the on-site microbiologist helped control consumption of banned agents.

This type of study suffers from the fact that cause-and-effect between HAI rates and changes in antibiotic consumption are notoriously difficult to prove [1]. Antimicrobial policy changes are confounded by infection control practices on the wards, including urinary and vascular catheter use $[16,17]$. In addition, there was occasional use of related drugs such as moxifloxacin, levofloxacin and cefuroxime, albeit at a low level, which could have influenced the effect of the policy. Meropenem consumption decreased 
throughout the study and could have had an impact on $C$. difficile rates, although there was no statistical correlation. We believe that this was because overall use of meropenem was low and, furthermore, it was generally only used for patients in intensive care.

Consumption of empirical amoxicillin and gentamicin escalated throughout the study and could also have confounded the overall effect. Given the surge in gentamicin prescribing, we were forced to monitor the management of dosage and levels, with auditing of incidents regarding gentamicin. After initial turbulence, gentamicin is now generally well managed, although resistance is increasing among hospital isolates (data not included). Amoxicillin resistance also increased among hospital $E$. coli isolates, although this has only recently been detected. Ecological studies are limited compared with individual-level analysis because is not possible to evaluate time as an important exposure variable related to the emergence of resistance. The ecological 'bias' or analysis of aggregated data may be compromised by the failure of level-effect estimates to reflect the biological effect at the individual patient level [18].

Whilst low rates of $C$. difficile have been maintained to date, it is possible that continued restrictions have influenced MRSA and ESBL-producing coliform acquisition longer term, subject to the confounders already described. Three years after initiating the antibiotic policy, the 2011 rate of hospital-acquired $C$. difficile was 0.259 cases $/ 1000$ pt-bds, for MRSA it was 0.409 cases $/ 1000$ pt-bds and for ESBL-producing coliforms it was 0.809 cases/1000 pt-bds. These rates are among the lowest achieved since the study began and reflect observed lower rates of surgical site infection.

Retrospective search of the database for carbapenem resistance among hospital-acquired ESBL-producing coliforms using appropriate clinical definitions identified five cases in 2008 , one in 2009 , three in 2010, one in 2011 and none so far during 2012. These numbers are too small to draw any conclusions, but it is possible that the restrictive policy has had some impact on extreme drug resistance in this hospital. Three of the cases in 2008 were fatal [19]. Whilst there have not been any specific interventions to control ESBLproducing coliforms during the last 4 years, universal screening for MRSA began in 2010 and continued throughout 2011. This may have contributed towards reduced hospital-acquired MRSA in 2011, since there was only an insignificant reduction in MRSA acquisition from 2007 to 2009 [5].

In conclusion, banning ceftriaxone and ciprofloxacin from firstline prescribing resulted in a rapid reduction in hospital-acquired C. difficile, with smaller reductions in ESBL-producing coliforms and MRSA over an initial 2-year period. Quinolone consumption appears to be driving ESBL-producing coliform acquisition in this hospital. Continued restrictions on antibiotic prescribing have helped maintain low rates of $C$. difficile to date. Infection control interventions aside, it is possible that the policy has also impacted upon ESBL-producing coliform and MRSA acquisition long term. Antimicrobial stewardship is fundamental for controlling HAIs and we should strive to reduce antibiotic use at all costs [20].

\section{Acknowledgments}

Frances Kerr, Catherine Irvine and Margaret Crookston (Pharmacy) helped with antimicrobial consumption data; nurses Christina Coulombe and Lorraine McWilliams helped collect and validate hospital-acquired infection data. The study could not have been performed without support from NHS Lanarkshire Antibiotic \& Infection Group and Hairmyres medical staff.

Funding: No funding sources.

Competing interests: Sole conflicts of interest are previous conference support for SJD from Janssen-Cilag, Pfizer and Novartis. Ethical approval: Not required.

\section{References}

[1] Hunter PA, Dawson S, French GL, Goossens H, Hawkey PM, Kuijper EJ, et al. Antimicrobial-resistant pathogens in animals and man: prescribing, practices and policies. J Antimicrob Chemother 2010;65(Suppl. 1):i3-17.

[2] Avorn JL, Barrett JF, Davey PG, McEwan SA, O’Brien TF, Levy SB. Antibiotic resistance: synthesis of recommendations by expert policy groups. Alliance for the Prudent Use of Antibiotics. Geneva, Switzerland: World Health Organization; 2001. p. 1-155. WHO/CDS/CSR/DRS/2001.10.

[3] Dancer SJ. The problem with cephalosporins. J Antimicrob Chemother 2001;48:463-78.

[4] Carling P, Fung T, Killion A, Terrin N, Barza M. Favorable impact of a multidisciplinary antibiotic management program conducted during 7 years. Infect Control Hosp Epidemiol 2003;24:699-706.

[5] Liebowitz LD, Blunt MC. Modification in prescribing practices for thirdgeneration cephalosporins and ciprofloxacin is associated with a reduction in meticillin-resistant Staphylococcus aureus bacteraemia rate. J Hosp Infect 2008;69:328-36.

[6] Health Protection Scotland. Report on review of Clostridium difficile associated disease cases and mortality in all acute hospitals in Scotland from December 2007-May 2008. Health Protection Scotland/National Services Scotland; 2008. http://www.documents.hps.scot.nhs.uk/hai/sshaip/publications/cdad/cdadreview-2008-07.pdf [accessed September 2012].

[7] Hensgens MPM, Goorhuis A, Dekkers OM, Kuijper EJ. Time interval of increased risk for Clostridium difficile infection after exposure to antibiotics. J Antimicrob Chemother 2012;67:742-8.

[8] Starr JM, Campbell A, Renshaw E, Poxton IR, Gibson GJ. Spatio-temporal stochastic modelling of Clostridium difficile. J Hosp Infect 2009;71:49-56.

[9] Davey P, Brown E, Fenelon L, Finch R, Gould I, Holmes A, et al. Systematic review of antimicrobial drug prescribing in hospitals. Emerg Infect Dis 2006;12:211-6.

[10] Nseir S, Di Pompeo C, Soubrier S, Delour P, Lenci H, Roussel-Delvallez M, et al. First-generation fluoroquinolone use and subsequent emergence of multiple drug-resistant bacteria in the intensive care unit. Crit Care Med 2005;33:283-9.

[11] Jacoby TS, Kuchenbecker RS, dos Santos RP, Magedanz L, Guzatto P, Moreira LB. Impact of hospital-wide infection rate, invasive procedures use and antimicrobial consumption on bacterial resistance inside an intensive care unit. J Hosp Infect 2010;75:23-7.

[12] Aldeyab MA, Harbarth S, Vernaz N, Kearney MP, Scott MG, Darwish Elhajji FW, et al. The impact of antibiotic use on the incidence and resistance pattern of extended-spectrum $\beta$-lactamase-producing bacteria in primary and secondary healthcare settings. Br J Clin Pharmacol 2012;74:171-9.

[13] Bal AM, Gould I. Antibiotic stewardship: overcoming implementation barriers. Curr Opin Infect Dis 2011;24:357-62.

[14] Soares-Weiser K, Brezis M, Tur-Kaspa R, Paul M, Yahav J, Leibovici L. Antibiotic prophylaxis for cirrhotic patients with gastrointestinal bleeding. Cochrane Database Syst Rev 2002:CD002907.

[15] Hand K. Antibiotic pharmacists in the ascendancy. J Antimicrob Chemother 2007;60(Suppl. 1):i73-6.

[16] Aldeyab MA, Hughes CM, Kearney MP, Scott MG, McDowell DA, Hanley J, et al. Comparison of the effect of ciprofloxacin and Tazocin on the incidence of meticillin-resistant Staphylococcus aureus (MRSA) in an intensive care unit. Int J Antimicrob Agents 2008;32:499-504.

[17] Friedmann R, Raveh D, Zartzer E, Rudensky B, Broide E, Attias D, et al Prospective evaluation of colonization with extended-spectrum $\beta$-lactamase (ESBL)-producing Enterobacteriaceae among patients at hospital admission and of subsequent colonization with ESBL-producing Enterobacteriaceae among patients during hospitalization. Infect Control Hosp Epidemiol 2009;30:534-42.

[18] Harbarth S, Samore MH. Interventions to control MRSA: high time for timeseries analysis? J Antimicrob Chemother 2008;62:431-3.

[19] Findlay J, Hamouda A, Dancer SJ, Amyes SG. Rapid acquisition of decreased carbapenem susceptibility in a strain of Klebsiella pneumoniae arising during meropenem therapy. Clin Microbiol Infect 2012;18:140-6.

[20] Gould I. Antibiotic resistance: understanding how to control it. Int J Antimicrob Agents 2012;40:193-5. 